\title{
Epidemiology of prostate cancer
}

\author{
Matthew R. Cooperberg ${ }^{1,2} \cdot$ June M. Chan ${ }^{1,2}$
}

Received: 10 April 2017 / Accepted: 18 April 2017 / Published online: 16 May 2017

(C) Springer-Verlag Berlin Heidelberg 2017

In the setting of a growing world population and economy, and slow but steady improvements in public health, the world's older male population continues to grow and age; trends which will only accelerate in the decades to come [1]. In this context, prostate cancer is a growing concern in global epidemiology. More than one million cases are diagnosed annually, and the mortality burden has risen to over 300,000 deaths per year. Prostate cancer has now surpassed esophageal cancer in terms of male mortality burden, and is the fifth leading cause of cancer death among men [2]. Both incidence and mortality vary tremendously across geographic regions and populations [2], almost certainly reflecting multifactorial impacts of genetic variation; diet, lifestyle, and environmental factors; access to care and overall life expectancy; and variations in use of prostate specific antigen-based screening policies.

In this special theme issue of the World Journal of Urology, we explore several aspects of prostate cancer global epidemiology in detail. Auvinen et al. (10.1007/s00345016-1953-9) provide an overview of variation in prostate cancer incidence at the national level based on GLOBOCAN data, focusing on associations with intensity of medical expenditures. Akaza et al. (10.1007/s00345-0161939-7) review the rapidly evolving epidemiology of the disease in East Asia, where both incidence and mortality have historically been low and management quite different

Matthew R. Cooperberg

matthew.cooperberg@ucsf.edu

1 Department of Urology, UCSF Helen Diller Family Comprehensive Cancer Center, 550 16th St, San Francisco, CA 94143, USA

2 Department of Epidemiology \& Biostatistics, UCSF Helen Diller Family Comprehensive Cancer Center, 550 16th St, San Francisco, CA 94143, USA than in most western countries. The literature on modifiable risk factors has continued to expand rapidly. Peisch et al. (10.1007/s00345-016-1914-3) review diet and lifestyle risk factors-focusing in particular on exercise and smoking cessation-as important determinants of both prostate cancer specific and overall health outcomes. Stopsack et al. (10.1007/s00345-016-1912-5) critique the literature on the effects of metformin, statins, and other medications on prostate cancer risk, and Albanes et al. (10.1007/ s00345-016-1878-3) provide a deep dive into the impacts of vitamins and other micronutrients. Finally, Nordström et al. (10.1007/s00345-016-1907-2) offer a commentary on one possible evolution in prostate cancer screening policy, based on the innovative STHLM-3 trial.

We hope these articles will be of great interest to the Journal's readership, and will help stimulate the next generation of research into the genetic and environmental determinants of prostate cancer risk and mortality. Through a better understanding of both how and why prostate cancer epidemiology varies so greatly, we will gain deeper insights into the disease's biology, to the eventual benefit of at-risk men around the world.

\section{References}

1. Economic UNDOE, Affairs S (2001) World Population Ageing: 1950-2050. 1-5

2. Torre LA, Bray F, Siegel RL et al (2015) Global cancer statistics, 2012. CA Cancer J Clin 65:87-108. doi:10.3322/caac. 21262 